\title{
Limited role of regulatory $T$ cells during acute Theiler virus-induced encephalitis in resistant C57BL/6 mice
}

\author{
Chittappen K Prajeeth ${ }^{1 \dagger}$, Andreas Beineke ${ }^{2,4+}$, Cut Dahlia Iskandar ${ }^{2,4}$, Viktoria Gudi ${ }^{1}$, Vanessa Herder ${ }^{2}$, \\ Ingo Gerhauser ${ }^{2}$, Verena Haist ${ }^{2}$, René Teich ${ }^{3}$, Jochen Huehn ${ }^{3}$, Wolfgang Baumgärtner ${ }^{2,4}$ and Martin Stangel ${ }^{1,4^{*}}$
}

\begin{abstract}
Background: Theiler's murine encephalomyelitis virus (TMEV) infection represents a commonly used infectious animal model to study various aspects of the pathogenesis of multiple sclerosis (MS). In susceptible SJL mice, dominant activity of Foxp $3^{+} \mathrm{CD}^{+}$regulatory $\mathrm{T}$ cells (Tregs) in the CNS partly contributes to viral persistence and progressive demyelination. On the other hand, resistant $\mathrm{C57BL} / 6$ mice rapidly clear the virus by mounting a strong antiviral immune response. However, very little is known about the role of Tregs in regulating antiviral responses during acute encephalitis in resistant mouse strains.
\end{abstract}

Methods: In this study, we used DEREG mice that express the diphtheria toxin (DT) receptor under control of the foxp3 locus to selectively deplete Foxp $3^{+}$Tregs by injection of DT prior to infection and studied the effect of Treg depletion on the course of acute Theiler's murine encephalomyelitis (TME).

Results: As expected, DEREG mice that are on a C57BL/6 background were resistant to TMEV infection and cleared the virus within days of infection, regardless of the presence or absence of Tregs. Nevertheless, in the absence of Tregs we observed priming of stronger effector $T$ cell responses in the periphery, which subsequently resulted in a transient increase in the frequency of IFNy-producing T cells in the brain at an early stage of infection. Histological and flow cytometric analysis revealed that this transiently increased frequency of brain-infiltrating IFNY-producing $T$ cells in Treg-depleted mice neither led to an augmented antiviral response nor enhanced inflammation-mediated tissue damage. Intriguingly, Treg depletion did not change the expression of IL-10 in the infected brain, which might play a role for dampening the inflammatory damage caused by the increased number of effector $T$ cells.

Conclusion: We therefore propose that unlike susceptible mice strains, interfering with the Treg compartment of resistant mice only has negligible effects on virus-induced pathologies in the CNS. Furthermore, in the absence of Tregs, local anti-inflammatory mechanisms might limit the extent of damage caused by strong anti-viral response in the CNS.

Keywords: Regulatory T cells, Theiler's virus, Interleukin-10

\footnotetext{
* Correspondence: stangel.martin@mh-hannover.de

${ }^{\dagger}$ Equal contributors

'Department of Neurology, Clinical Neuroimmunology and Neurochemistry, Hannover Medical School, Carl-Neuberg-Str. 1, Hannover 30625, Germany

${ }^{4}$ Center of Systems Neuroscience, Hannover, Germany

Full list of author information is available at the end of the article
} 


\section{Background}

Multiple sclerosis (MS), one of the most frequent central nervous system (CNS) diseases in young adults, is a chronic demyelinating disease of unknown etiology and probably multifactorial causes. Based on the generation of myelin-specific immune responses, MS is regarded as an autoimmune disease [1,2], possibly triggered by virus infections [3,4]. Due to clinical and pathological similarities, Theiler's murine encephalomyelitis (TME) represents a commonly used infectious animal model for the chronic-progressive form of MS in humans [5]. Here, intracerebral infection of mice with the low virulent BeAn-strain of the Theiler's murine encephalomyelitis virus (TMEV) causes an acute transient polioencephalitis [6], characterized by the infiltration of virus-specific lymphocytes [7]. Inadequate viral clearance in SJL mice leads to persistent infection of the CNS and immunemediated spinal cord demyelination [6,8-10]. In contrast, resistant C57BL/6 mice eliminate the virus from the CNS by specific cellular immunity, including effective $\mathrm{CD} 4^{+}$ and $\mathrm{CD}^{+} \mathrm{T}$ cell responses during the acute infection phase [11].

Regulatory T cells (Tregs), characterized by expression of the transcription factor forkhead box P3 (Foxp3), play a key role in the maintenance of immunological tolerance and prevent immunopathology [12-17]. However, in viral diseases Tregs can exhibit both beneficial effects by reducing immune-mediated tissue damage and detrimental effects due to their immunosuppressive properties, causing disease exacerbation or viral persistence, respectively. Recently, rapid expansion of Tregs associated with an increased expression of the immunosuppressive cytokine IL-10 has been demonstrated in the brain of susceptible SJL mice but not in resistant C57BL/6 mice following TMEV infection $[11,18]$. Moreover, functional inactivation of Tregs by anti-CD25 antibodies prior to infection results in an enhanced virus-specific immunity, reduced viral load, and delayed disease progression, while the adoptive transfer of Tregs leads to disease exacerbation in TMEVinfected SJL mice $[11,19]$. However, these treatments have failed to influence the disease course in resistant mice strains $(\mathrm{C} 57 \mathrm{BL} / 6)$, demonstrating the complexity of protective immune responses in infectious CNS disorders.

In order to obtain further insights into the relevance of Tregs for disease resistance and antiviral immunity in TME, the kinetics of CNS-infiltrating immune cells and the underlying chemokine and cytokine expression following selective ablation of Foxp $3^{+}$Treg in C57BL/6 mice was investigated.

\section{Methods \\ Mice}

BAC-transgenic DEREG mice have been described previously [20]. All animals were gender matched and control mice used in our experiments were non-transgenic littermates obtained from DEREG breeding. Mice were bred and maintained under specific pathogen-free condition in our animal facilities. All animals used in experiments were 8 weeks of age. The animal experiments were approved and authorized by the local authorities (Niedersächsisches Landesamt für Verbraucherschutz- und Lebensmittelsicherheit (LAVES), Oldenburg, Germany, permission number 33.12-42502-04-10/0150) and performed according to international guidelines on the use of laboratory animals [21].

\section{DT treatment}

DEREG and WT control mice received $1 \mu \mathrm{g}$ of diphtheria toxin (DT) intraperitoneally (ip) (Calbiochem, San Diego, CA, USA) in $100 \mu \mathrm{l}$ PBS on days -2 and -1 corresponding to the infection.

\section{Intracerebral injection}

Eight-week-old male DEREG mice and non-transgenic littermate controls were inoculated into the right hemisphere with $1.63 \times 10^{6}$ plaque-forming units/mouse of the BeAn strain of TMEV in $20 \mu \mathrm{l}$ DMEM (PAA Laboratories, Cölbe, Germany) with $2 \%$ fetal calf serum and $50 \mu \mathrm{g} / \mathrm{kg}$ gentamicin. Inoculation was carried out under general anesthesia with medetomidine $(0.5 \mathrm{mg} / \mathrm{kg}$, Domitor, Pfizer, Karlsruhe, Germany) and ketamine $(100 \mathrm{mg} / \mathrm{kg}$, ketamine 10\%, WDT eG, Garbsen, Germany).

\section{Isolation of cells from brain}

Mice were transcardially perfused with PBS and the brains were carefully dissected and collected in Hank's balanced salt solution containing $15 \mathrm{mM}$ HEPES and 5\% glucose. The brain was cut into small pieces and digestion was carried out by incubating the tissue fragments in DMEM containing $0.5 \mathrm{mg} / \mathrm{ml}$ collagenase D (Roche, Mannheim, Germany) and $10 \mu \mathrm{g} / \mathrm{ml}$ DNase I (Roche, Mannheim, Germany) in an orbital shaker at $80 \mathrm{rpm}$ for 30 minutes at $37^{\circ} \mathrm{C}$. Tissue suspensions were then passed through a 70$\mu \mathrm{m}$ mesh, pelleted, resuspended in a $40 \%$ isotonic Percoll solution (GE Healthcare, Uppsala, Sweden), and layered over 70\% isotonic Percoll solution. After centrifugation at 2,100 rpm for 30 minutes at room temperature, the upper myelin layer was aspirated and the cells at the interface were collected, washed once with PBS and then used for further analyses.

\section{Antibodies and reagents}

Fluorochrome conjugated antibodies specific for mouse, anti-CD3 (clone: 145-2C11), anti-CD4 (clone: RM4-5), anti-Foxp3 (clone: MF-14), anti-IFNY (clone: XMG1.2) and anti-IL-17A (clone: TC11-18H10.1) were all purchased from Biolegend, San Diego, CA, USA. Anti-CD8 
(clone: 53-6.7) was purchased from eBioscience, Frankfurt, Germany.

\section{Flow cytometry}

For cell surface staining, cells were preincubated with CD16/32 Fc-block antibody (clone: 93, eBioscience, Frankfurt, Germany) at room temperature (RT) for $10 \mathrm{mi}-$ nutes, and subsequently incubated with the indicated conjugated antibody cocktail for 30 minutes in a total volume of $100 \mu \mathrm{l}$ of PBS containing 1\% FBS. For live/dead staining, cells were incubated in fixable viability dye eFluor ${ }^{\circ}$ 506 (1:1,000 dilution; eBioscience, Frankfurt, Germany) at $4^{\circ} \mathrm{C}$ for 30 minutes. For intracellular cytokine staining, cells were restimulated for 6 hours in complete DMEM (Gibco, Darmstard, Germany) in the presence of $50 \mathrm{ng} / \mathrm{ml}$ phorbol 12-myristate 13-acetate and $500 \mathrm{ng} / \mathrm{ml}$ ionomycin (both from Sigma-Aldrich, St. Louis, MO, USA). For the last 4 hours, brefeldin A $(10 \mu \mathrm{g} / \mathrm{ml}$; Sigma-Aldrich, St. Louis, MO, USA) was added to the cultures. Intracellular staining/transcription factor was performed using Foxp3 staining buffer set (eBioscience, Frankfurt, Germany) according to the manufacturers' recommendations. Samples were acquired on LSR II (BD Biosciences, San Jose, CA, USA) and analyzed with FlowJo software (Tree Star, Ashland, OR, USA).

\section{Histology, immunohistochemistry and immunofluorescence}

For histology and immunohistochemistry the cerebrum was fixed in $10 \%$ formalin for 24 hours, embedded in paraffin wax and stained with $\mathrm{H}$ \& $\mathrm{E}$. Inflammatory responses were graded based upon the degree of perivascular infiltrates (PVI) using a semiquantitative scoring system: $1=1$ to 25 cells (mild); $2=26$ to 50 cells (moderate); $3=>50$ cells per high power field (severe) as previously described [22]. Immunohistochemistry was performed using a polyclonal rabbit anti-CD3 antibody (DakoCytomation, Hamburg, Germany) for the detection of $\mathrm{T}$ cells. For blocking of the endogenous peroxidase, formalin-fixed, paraffin-embedded tissue sections were treated with $0.5 \%$ $\mathrm{H}_{2} \mathrm{O}_{2}$ diluted in methanol for 30 minutes at RT. Antigen retrieval was done by heating the sections in $10 \mathrm{mM}$ Na-citrate buffer $\mathrm{pH} 6.0$ for 20 minutes in a microwave oven $(800 \mathrm{~W})$. Subsequently, slides were incubated with the respective primary antibody overnight at $4^{\circ} \mathrm{C}$. Biotinylated goat-anti-rabbit IgG diluted 1:200 (Vector Laboratories, Burlingame, CA, USA) was used as a secondary antibody. Sections used as negative controls for CD3 immunohistochemistry were incubated with rabbit normal serum at a dilution of 1:2,000 (Sigma-Aldrich Chemie $\mathrm{GmbH}$, Taufkirchen, Germany). Slides were subsequently incubated with the peroxidase-conjugated avidin-biotin complex (ABC method, Vector Laboratories, Burlingame, CA, USA) for 30 minutes at RT. After the positive antigen-antibody reaction visualization by incubation with 3.3-diaminobenzidine-tetrachloride (DAB) in $0.1 \mathrm{M}$ imidazole, sections were counterstained with Mayer's hematoxylin.

For immunofluorescence staining, antigen retrieval was performed as mentioned above, following which the sections were blocked with $10 \%$ normal donkey serum. Slides were incubated with primary anti-IL10 (clone: M-18: Santa Cruz Biotechnology, Santa Cruz, CA, USA) along with anti-CD3, anti-glial fibrillary acidic protein (GFAP) (DakoCytomation, Hamburg, Germany) or anti-Iba1 (Wako, Osaka, Japan) antibodies for 2 hours at RT and after thorough washing the slides were incubated with fluorochrome conjugated secondary donkey anti-goat antibodies for 1 hour.

The density of positive cells $/ \mathrm{mm}^{2}$ in the cerebral neuroparenchyma was manually counted in randomly selected cerebral areas with a 40-fold objective and a 10 -fold calibrated eyepiece reticule (Olympus Europe, Hamburg, Germany).

\section{RNA isolation}

RNA was isolated from 10 to $40 \mathrm{mg}$ tissue of each frozen brain using an Omni's PCR Tissue Homogenizing Kit (Süd-Laborbedarf GmbH, Gauting, Germany) and RNeasy ${ }^{\circledast}$ Lipid Tissue Mini Kit (\#74804; Qiagen GmbH, Hilden, Germany).

\section{Reverse transcription-polymerase chain reaction (RT-PCR)} Equal amounts of RNA were subsequently transcribed into cDNA with the High Capacity cDNA Reverse Transcription Kit with RNase Inhibitor (\#4374966; Applied Biosystems ${ }^{\oplus}$; Life Technologies GmbH, Darmstadt, Germany). RT-PCR for the quantification of TMEV (forward primer: 5'-GACTAATCAGAGGAACGTCAGC-3'; reverse primer: 5'-GTGAAGAGCGGCAAGTGAGA-3') and three housekeeping genes: glyceraldehyde 3-phosphate dehydrogenase (GAPDH; forward primer: 5'-GAGGC CGGTGCTGAGTATGT-3'; reverse primer: 5'-GGTG GCAGTGATGGCATGGA-3'), $\beta$-actin (forward primer: 5'-GGCTACAGCTTCACCACCAC-3'; reverse primer: 5'-ATGCCACAGGATTCCATACC-3'), hypoxanthineguanine phosphoribosyltransferase (HPRT; forward primer: 5'-GGACCTCTCGAAGTGTTGGA-3'; reverse primer: 5'-TTGCGCTCATCTTAGGCTTT-3') in brain tissues was performed using the Mx3005 $\mathrm{P}^{\mathrm{TM}}$ Multiplex Quantitative PCR System (Agilent Technologies Deutschland GmbH, Böblingen, Germany) and $\mathrm{SYBR}^{\odot}$ Green I as DNA-binding dye as described [23,24]. Ten-fold serial dilution standards ranging from $10^{8}$ to $10^{2}$ copies $/ \mu \mathrm{l}$ were used to quantify the results. A normalization factor achieved from the three housekeeping genes was calculated using the geNorm software version 3.4 to correct for 
experimental variations [25]. Specificity of each reaction was controlled by melting curve analysis.

For other gene expression analysis, quantitative real-time PCR was performed using the StepOne ${ }^{\mathrm{TM}}$ Real-Time PCR System and appropriate TaqMan probes (Applied Biosystems, Life Technologies GmbH, Darmstadt, Germany, Additional file 1: Table S1). The $\Delta \Delta \mathrm{Ct}$ method was applied to determine differences in the expression of CCL2, CXCL10, interleukin-6 (IL-6), interleukin-10 (IL-10), inferferon- $\gamma($ IFN $\gamma)$, tumor necrosis factor- $\alpha$ (TNF- $\alpha)$ and inducible NO synthase (NOS2) and granulocyte-macrophage colony stimulating factor (GM-CSF) between uninfected and TMEV-infected animals that were previously treated with PBS or DT. Changes in mRNA expression levels were calculated after normalization to the arithmetic mean of HPRT and GAPDH.

\section{Statistical analysis}

All statistical analyses were conducted using GraphPad Prism 5.0 (GraphPad Software, La Jolla, CA, USA). All data are expressed as group mean \pm SD unless otherwise stated. The data generated from several mice from each group was analyzed using Student's $t$-test. Results were considered statistically significant at $P<0.05$.

\section{Results}

Treg depletion prior to TMEV infection enhances the infiltration of $\mathrm{T}$ cells into the brain

In our initial attempts we assessed the outcome of Treg depletion on the acute response to TMEV infection. For this we employed the strategy of depleting Foxp3 ${ }^{+}$Treg prior to TMEV infection by ip administration of DT into DEREG mice, which express the DT receptor under the control of the foxp3 locus [20]. Analysis of blood 1 day after DT injection revealed that most of the Foxp3 $3^{+} \mathrm{CD}_{4}^{+}$ $\mathrm{T}$ cells were depleted from circulation (Additional file 2: Figure S1). Following intracerebral inoculation of the BeAn strain of TMEV into Treg-depleted and nondepleted mice, the inflammatory response and virus load were assessed. Histology revealed no differences in the degree of neuroinflammation between Treg-depleted and non-depleted mice (data not shown). Interestingly, despite the similar severity of encephalitis among the groups, we observed in immunohistochemistry a significantly higher number of $\mathrm{CD}^{+} \mathrm{T}$ cells in the cerebrum of Treg-depleted mice at 3 days post inoculation (dpi) "However, this difference in the number of $\mathrm{T}$ cells was transient and was not observed at $7 \mathrm{dpi}$ (Figure 1A and B). Such differences were not evident among PBS- and DT-treated wild type (WT) littermate controls at these time points, thus confirming that transient increase in the number of T cells was the result of Treg depletion (Additional file 3: Figure S2A). These results suggest that Tregs might regulate the early recruitment of effector $\mathrm{T}$ cells to the sites of infection.

\section{Predominantly IFNY-producing T cells infiltrate into the brain}

Our next attempt was to assess the effect of Treg depletion on the antiviral immune response in the CNS. IFNy is a prominent effector molecule in antiviral immune defense and produced mainly by effector lymphocyte populations. Measuring the gene expression in infected brains by RT-PCR, we found, at $3 \mathrm{dpi}$, a 2 -fold higher expression of IFN $\gamma$ in Treg-depleted mice compared to non-depleted mice. No such differences in the expression of IFN $\gamma$ were observed at $7 \mathrm{dpi}$ between the two groups (Figure 2A). This coincides with the above mentioned observation where we found a transient increase of $\mathrm{T}$ cells at $3 \mathrm{dpi}$ in the absence of Tregs. Therefore, we sought to isolate immune cells from the brain of infected mice and analyze the $\mathrm{T}$ cell subpopulations in terms of their effector cytokine secretion profile. At $3 \mathrm{dpi}$, only few $\mathrm{T}$ cells were detected among the cells harvested from the brain of mice with an unaltered Treg compartment. Upon Treg depletion prior to infection there was an increased infiltration of $\mathrm{T}$ cells as we observed an increased frequency of $\mathrm{CD}^{+}$and $\mathrm{CD}^{+} \mathrm{T}$ cells in the brain (Additional file 4: Figure S3). Further supporting our RT-PCR data, flow cytometric analysis revealed a significantly higher percentage of IFN $\gamma$-producing Th1 and $\mathrm{CD}^{+}{ }^{+} \mathrm{T}$ cells among the cells isolated from the brain of Treg-depleted mice at 3 dpi but not at $7 \mathrm{dpi}$ (Figure $2 \mathrm{~B}$ and C). No IL-17producing $\mathrm{CD}^{+}$Th17 cells were found at both time points analyzed, regardless of the Treg status (Figure 2B). It is noteworthy that frequencies of IFN $\gamma$-producing $\mathrm{T}$ cells in the spleen of Treg-depleted mice were significantly higher at $3 \mathrm{dpi}$ and only marginally higher at $7 \mathrm{dpi}$ compared to non-depleted mice (Additional file 5: Figure S4). Further, we could confirm that the observed effects were only due to Treg depletion in DEREG mice, as no such differences were observed in DT-treated WT littermate controls (Additional file 3: Figure S2B-E). Taken together, these results suggest that a strong IFN $\gamma$-producing $\mathrm{T}$ cell response is primed in the periphery in the absence of Tregs and this might lead to an early recruitment of these effector T cells into the brain.

\section{Depletion of Tregs prior to infection does not influence viral clearance in DEREG mice}

Since we observed a higher frequency of IFN $\gamma$-producing $\mathrm{CD}^{+}$and $\mathrm{CD}^{+} \mathrm{T}$ cells infiltrating into the brain of TMEV-infected mice in the absence of Tregs at $3 \mathrm{dpi}$, we expected an enhanced and faster viral clearance in Tregdepleted mice. Therefore, we sought to assess the viral load by RT-PCR in the brain at 3 dpi and 7 dpi. Significantly lower copy numbers of viral RNA were detected at $7 \mathrm{dpi}$ compared to $3 \mathrm{dpi}$, indicating a potent antiviral response in TMEV-infected mice. However, it appears that Treg depletion had no impact on the antiviral response as 


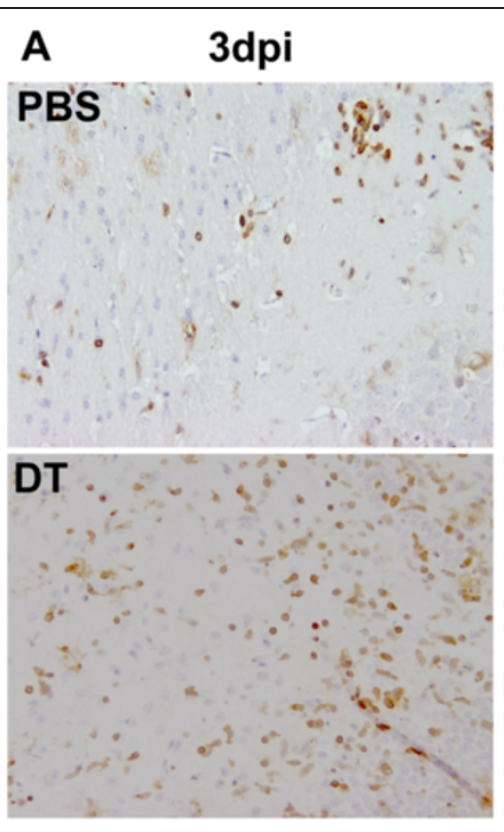

B

3dpi

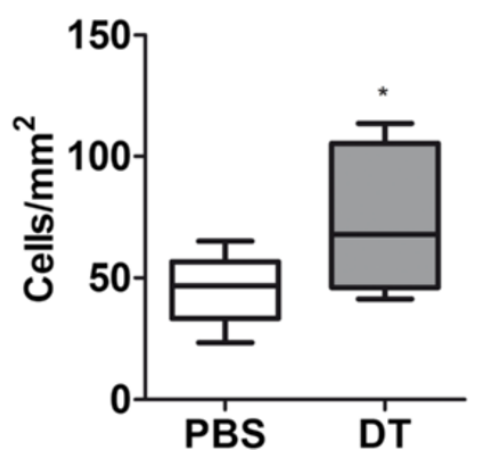

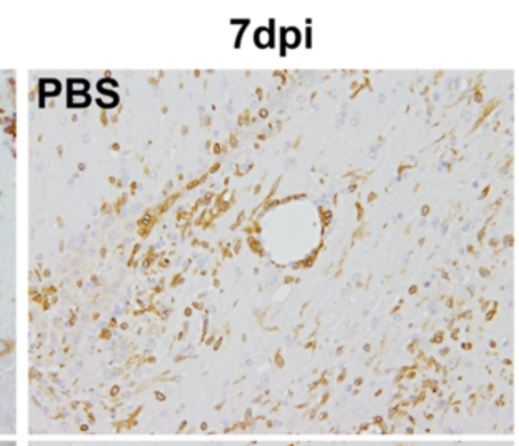

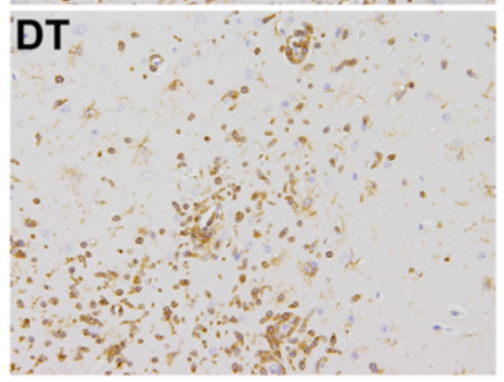

7dpi

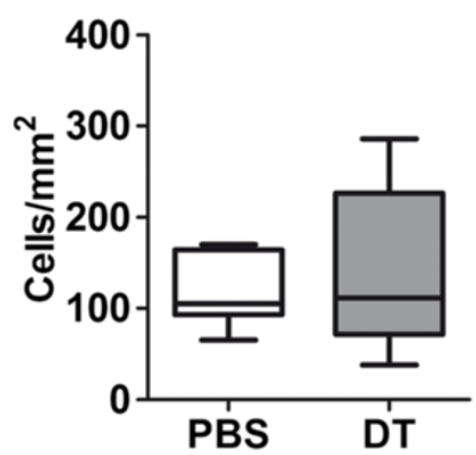

Figure 1 Early recruitment of T cells into Theiler's murine encephalomyelitis virus (TMEV)-infected brain in the absence of Tregs. Following intraperitoneal administration of PBS or diphtheria toxin (DT), DEREG mice were intracerebrally infected with TMEV. (A) Immunohistochemistry of TMEV infected brains at 3 days post inoculation (dpi) (left panel) and 7 dpi (right panel) reveals higher numbers of CD3 ${ }^{+} T$ cells only at 3 dpi in DT-treated mice (lower panel) compared to PBS-treated mice (upper panel). (B) Quantification of CD3 ${ }^{+} T$ cells in the cerebral neuroparenchyma of 6 to 8 infected mice reveals a significantly increased number on T cells in DT-treated mice at 3 dpi. Box and whisker plots display median and quartiles with maximum and minimum values. ${ }^{*} P$-value $<0.05$.

we did not observe any significant differences in the viral load between Treg-depleted and non-depleted mice neither at $3 \mathrm{dpi}$ nor at $7 \mathrm{dpi}$ (Figure 3).

\section{Treg depletion did not alter the expression of inflammatory mediators in the brain}

It was rather puzzling to have an increased frequency of IFN $\gamma^{+} \mathrm{T}$ cells and yet not to observe any difference in the viral load or tissue damage. Therefore, we studied the local inflammatory response triggered by TMEV infection in the absence of Tregs. We compared gene expression of certain inflammatory mediators in response to TMEV infection in the brain of Treg-depleted and non-depleted mice. Chemokines CCL2 and CXCL10, believed to be produced by resident glial cells and known to play a major role in the recruitment of monocytes and T cells from the periphery, were strongly induced upon TMEV infection (Figure 4A and B). Nevertheless, depletion of Tregs had no influence on their expression both at $3 \mathrm{dpi}$ and $7 \mathrm{dpi}$. Similarly, a potent pro-inflammatory response was induced upon TMEV infection characterized by significantly increased expression of GM-CSF, IL-6, TNF- $\alpha$, and NOS2 in these mice compared to uninfected controls. However, we did not detect any significant difference in the transcription of these genes between Treg-depleted and nondepleted mice (Figure 4A and B). 

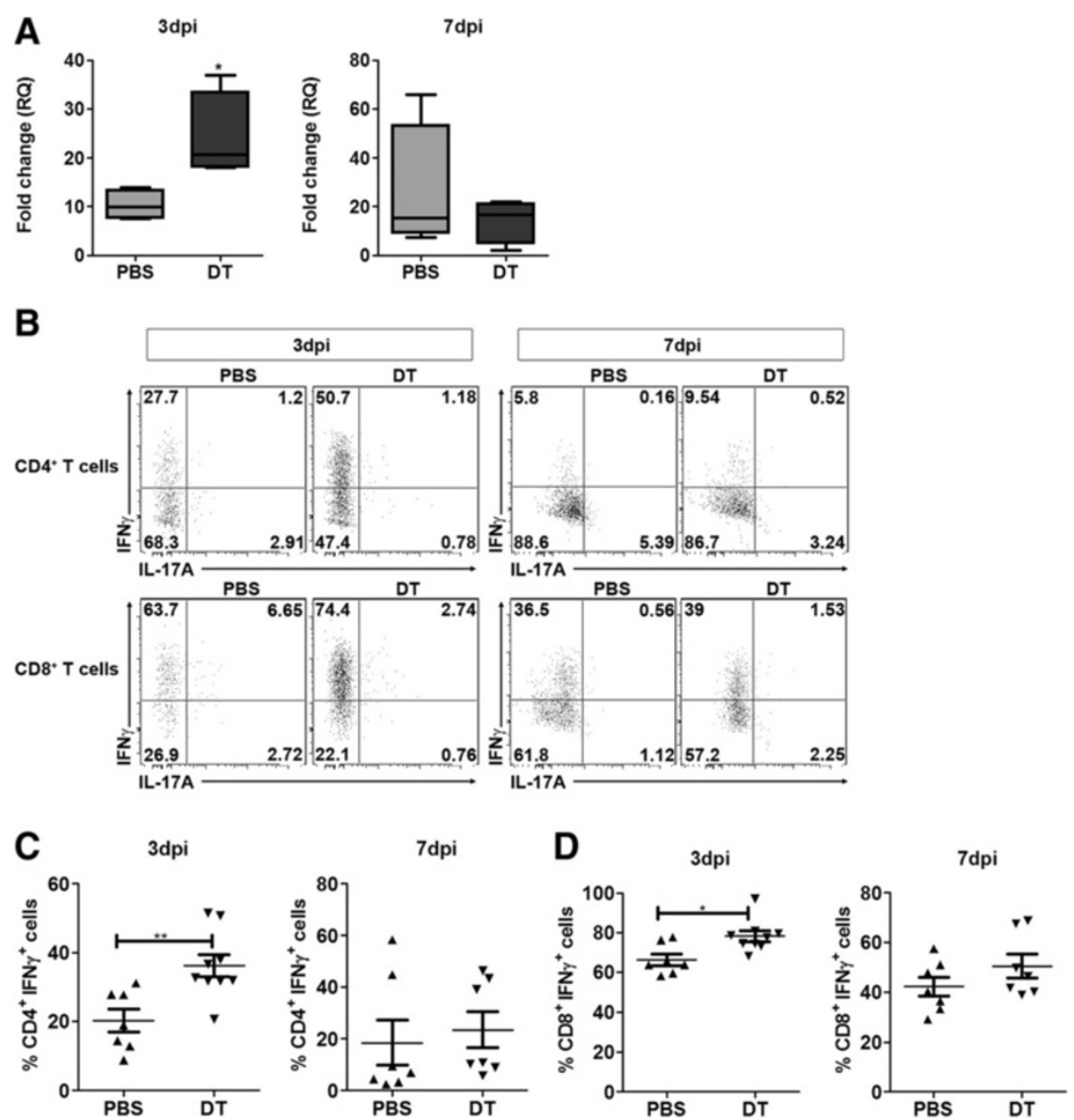

Figure 2 Increased frequency of IFNY-producing T cells found in the brain tissue of Treg-depleted mice post Theiler's murine encephalomyelitis virus (TMEV) infection. (A) RT-PCR of mRNA isolated from the brain of TMEV-infected DEREG mice treated with PBS or diphtheria toxin (DT) show an approximately 2-fold higher expression of IFNy in Treg-depleted (DT) mice compared to non-depleted mice (PBS) at 3 days post inoculation (dpi). However, no significant difference can be observed at $7 \mathrm{dpi}$. The graph represents mean \pm SD of the data obtained from four infected mice from each group. (B) Flow cytometry analysis of cells isolated from infected brains show a higher frequency of IFNY-producing T cells $\mathrm{CD}^{+}$(upper panel) and CD8 $8^{+}$(lower panel) T cells at $3 \mathrm{dpi}$ (left panel) and not at $7 \mathrm{dpi}$ (right panel). No IL-17-producing cells were found in any of the groups. Data obtained by analyzing the $\mathrm{CD}^{+} \mathrm{CD} 4^{+}$(C) and $\mathrm{CD}^{+} \mathrm{CD}^{+}$cells (D) from 7 to 9 infected mice from each group is shown in this plot. ${ }^{*} P$-value $<0.05,{ }^{*} P$-value $<0.01$.

\section{IL-10 produced by microglia/macrophage compensates for the lack of Tregs}

Another interesting observation from the RT-PCR analysis was that the expression of the anti-inflammatory cytokine IL-10 in the brain of infected mice was unaffected despite of Treg-depletion. The amount of IL-10 expressed was similar in Treg-depleted and nondepleted mice both at $3 \mathrm{dpi}$ and $7 \mathrm{dpi}$ (Figure 5A). However, expression of IL-10 was increased by few folds at $7 \mathrm{dpi}$, suggesting a role of locally produced IL-10 in neuroprotection. In an attempt to delineate the source of IL-10 we performed immunofluorescence co-stainings of $7 \mathrm{dpi}$ brain sections [18]. We found that the majority of IL-10 signals co-localized with Iba- $1^{+}$cells, indicating that microglia or infiltrating macrophages could be the source of IL-10 (Figure 5B). At the same time IL-10 was found lacking among the majority of $\mathrm{CD}^{+} \mathrm{T}$ cells and $\mathrm{GFAP}^{+}$astrocytes. However, we failed to detect any significant difference in the number of $\mathrm{Iba}^{+} \mathrm{IL}^{-10^{+}}$cells between PBS- and DT-treated groups at this time point (data not shown). Taken together these results suggest that IL-10 produced by microglia/macrophages compensates for the lack of Tregs and might limit the extent of tissue damage resulting from increased infiltration of effector $\mathrm{T}$ cells. 


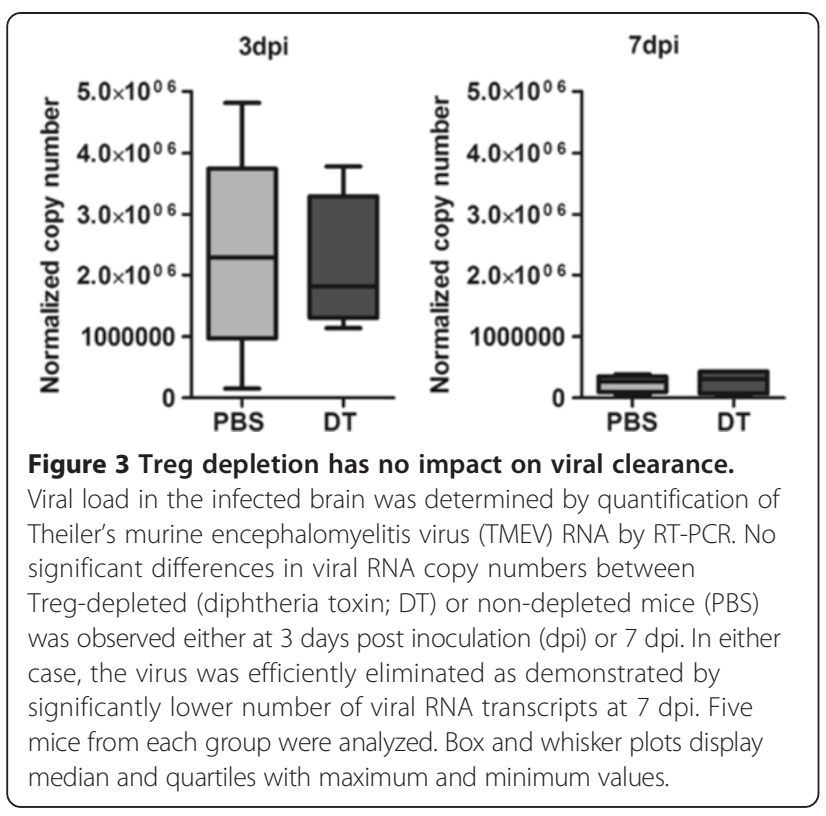

\section{Discussion}

In this study, we investigated the role of Foxp $3^{+}$Tregs during an acute viral infection in the CNS using DEREG mice in which Tregs can be selectively depleted by injecting DT. Our findings support the hypothesis that Tregs have no major influence in regulating immune responses against TMEV in the CNS of resistant C57BL/6 mice. However, this does not reflect an altogether null effect of Treg depletion as we did observe a transient increase in the number of effector Th1 and CD8 ${ }^{+} \mathrm{T}$ cells in the brain at an early stage of infection.

TME is a good model to study the pathogenesis of virus-mediated demyelinating diseases. In response to TMEV infection, $\mathrm{CD} 4^{+}$and $\mathrm{CD}^{+} \mathrm{T}$ cells are primed in the periphery, which subsequently infiltrate into the CNS and mount antiviral immune responses, leading to effective viral clearance within the first weeks of infection in resistant C57BL/6 mice. On the contrary, susceptible SJL mice show an inadequate viral clearance, which causes virus persistence and chronic demyelination [26,27]. One of several factors contributing to this phenomenon in SJL mice is the suppressive activity of infiltrating Tregs in the CNS together with insufficient effector $\mathrm{CD}^{+} \mathrm{T}$ cell responses $[11,28]$. It has been demonstrated that functional inactivation of Tregs prior to TMEV infection enhances viral clearance by augmenting $\mathrm{CD} 8^{+} \mathrm{T}$ cells, Th1 cells and antibody responses leading to delayed disease onset and progression [11]. These results suggest that Tregs lead to TMEV persistence by suppressing antiviral responses in mice with a susceptible background. On the other side, while inhibiting effector $\mathrm{T}$ cell function, Tregs eventually limit CNS inflammation and tissue damage. It has been shown by a previous study that Treg depletion prior to EAE induction increases disease severity [29]. Therefore, we intended to study the role of Tregs in resistant C57BL/ 6 mice, which mount an efficient TMEV-specific immunity. Interestingly, at $3 \mathrm{dpi}$ we observed a significantly higher frequency of IFNy-producing $\mathrm{CD}^{+}$and $\mathrm{CD}^{+} \mathrm{T}$ cells in the brain in the absence of Tregs. In contrast to findings in SJL mice, we did not observe any differences in the viral load between Treg-depleted and non-depleted mice suggesting that the anti-TMEV response in C57BL/6 mice is unaffected by the presence or absence of Tregs. This is in line with a recent report that used an alternative approach of adoptive transfer of in vitro-induced Tregs (iTreg) and found that early iTreg treatment exacerbated TMEV-induced demyelination in SJL mice while C57BL/6 mice were largely unaffected by such treatments [19]. In C57BL/6 mice, TMEV infection results in rapid expansion
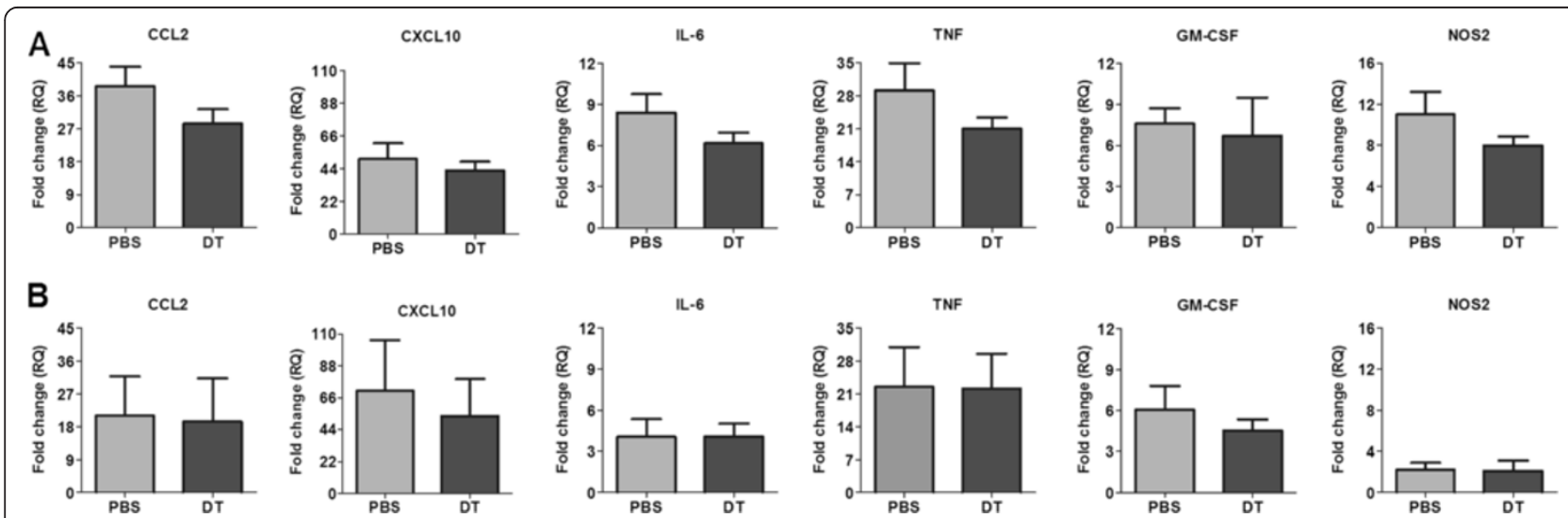

Figure 4 Expression of pro-inflammatory mediators is unaffected by Treg depletion. To study the local inflammatory response the gene expression of selected pro-inflammatory mediators (CCL2, CXCL10, GM-CSF, TNF-a, IL-6 and NOS2) was compared between Treg-depleted (diphtheria toxin; DT) and non-depleted (PBS) TMEV-infected mice at 3 days post inoculation (dpi) (A) and 7 dpi (B) by RT-PCR. Fold changes (RQ) was calculated by using $\Delta \Delta C$ t method (normalizing the $\Delta \mathrm{Ct}$ values obtained from infected mice to that of uninfected controls). The bar graphs represent mean $\pm \mathrm{SD}$ of fold change in gene expression obtained by analyzing four mice from each group. 

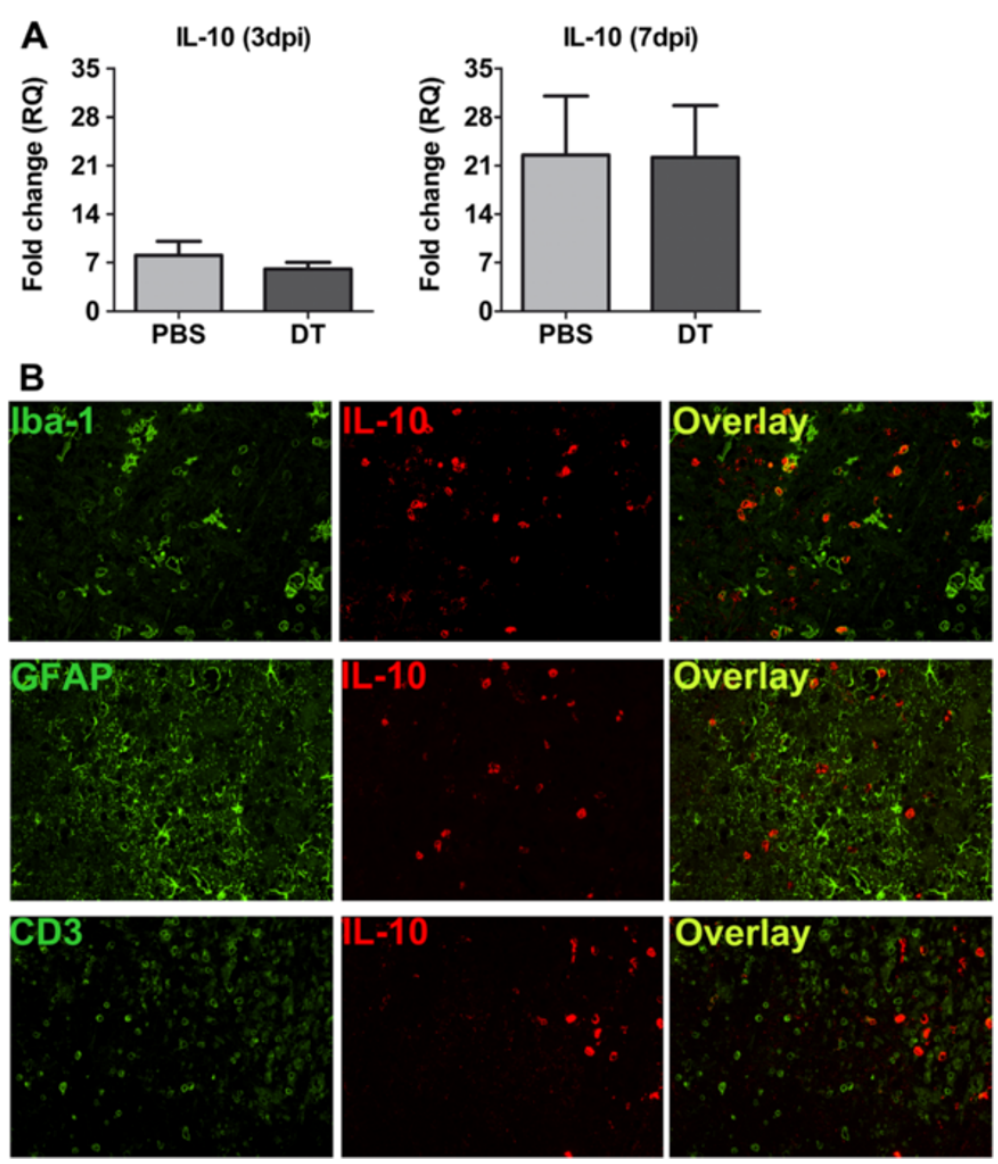

Figure 5 Locally produced IL-10 might be responsible for anti-inflammatory response. (A) IL-10 gene expression at 3 days post inoculation (dpi) and 7 dpi in Treg-depleted (diphtheria toxin; DT) and non-depleted (PBS) mice was assessed by RT-PCR. The bar graphs represent mean \pm SD of fold change in gene expression obtained by analyzing four mice from each group. (B) Double-immunofluorescence stainings of coronal brain sections using antibodies against IL-10 (red) in combination with Iba-1 (microglia), glial fibrillary acidic protein (GFAP) (astrocytes) or CD3 ${ }^{+}$( $T$ cells) at 7 dpi in Treg-depleted mice show that microglia are the major source of IL-10.

of effector $\mathrm{CD}^{+}$and $\mathrm{CD}^{+}{ }^{+} \mathrm{T}$ cells, which are efficient in clearing the virus [26]. Therefore, it might not be surprising that a mere increase in $\mathrm{T}$ cell numbers would lead to significant differences in viral load. It should be noted that the CNS has a limited regenerative capacity and hence an unwarranted inflammation triggered by immune cells infiltrating from the periphery might result in CNS tissue damage. Early studies have shown that an increase in proinflammatory Th1 responses enhances the development of demyelinating disease in resistant as well as in susceptible mice following TMEV infection [30,31]. Hence, we expected that a heightened IFN $\gamma$ response in the brain would trigger a stronger inflammatory response and cause more severe tissue damage. However, we neither observed early clinical signs in any of the groups nor any differences in the degree of tissue damage between Treg-depleted and non-depleted mice. This, we believe, is due to the fact that the inflammatory response in the brain is largely unaltered despite Treg depletion. Accordingly, the pro-inflammatory factors TNF- $\alpha$, GM-CSF, IL-6 and NOS2 displayed similar expression levels in mice with unaltered or reduced amounts of Tregs in response to TMEV. Another striking observation was that the expression of the antiinflammatory cytokine IL-10 was unaffected despite Treg depletion. This is interesting considering the fact that Tregs are an important source of IL-10 during CNS inflammation [29]. A recent study demonstrated that the expression of IL-10 during TMEV infection is mostly restricted to $\mathrm{T}$ cells; however, a portion of macrophages/ microglia also contributes to IL-10 expression in the brain of acutely TMEV-infected mice [18]. We observed expression of IL-10 as early as at $3 \mathrm{dpi}$ and was further increased at 7 dpi regardless of the presence or absence of Tregs. It is known that IL-10 has a dampening effect on inflammatory responses and plays a major role in inducing antiinflammatory mechanisms in the brain [19]. In C57BL/6 mice, resident glial cells, especially M2-type microglia/ macrophages, by producing IL-10 either in response to virus or due to heightened inflammatory response, might compensate for the lack of Tregs. Hence, we did not 
observe any damaging effects of additional effector $\mathrm{T}$ cells that infiltrate into the brain.

In conclusion, we could confirm that resistance to TMEV infection in C57BL/6 mice is largely due to the induction of effective $\mathrm{CD}^{+}$and $\mathrm{CD} 8^{+} \mathrm{T}$ cell responses, which is not influenced by Treg depletion. Furthermore, sustained expression of IL-10, mainly by resident glial cells during early infection, might limit the extent of damage caused by an unwanted immune response in the brain. While we believe that regulation by Tregs is straindependent, a significant role of local anti-inflammatory cytokines like IL-10 in neuroprotection during acute viral infections of the CNS cannot be ignored.

\section{Additional files}

Additional file 1: Table S1. Taqman probes used for RT-PCR analysis. Additional file 2: Figure S1. Depletion efficiency in DEREG mice post DT treatment. Wild type (WT) and DEREG mice were treated ip with two doses of $1 \mu \mathrm{g} / \mathrm{ml}$ of DT or with PBS alone on consecutive days. One day later, percentage of $\mathrm{CD}^{+} \mathrm{Foxp}^{+}$cells in the blood was determined by flow cytometry. The majority of Tregs were depleted in DEREG mice treated with DT whereas Tregs in WT mice were unaffected. Six mice were analyzed from each group. ${ }^{* * *}$-value $<0.001$.

Additional file 3: Figure S2. Diphtheria toxin administration in to WT mice has no obvious effects. In parallel to DEREG infection, non-transgenic littermates $(\mathrm{WT})$ treated with PBS and DT were infected with TMEV to test the off-target effects of DT. (A) Immunohistochemistry of coronal brain sections at $3 \mathrm{dpi}$ (left panel) and $7 \mathrm{dpi}$ (right panel) to detect $\mathrm{CD}^{+}{ }^{+} \mathrm{T}$ cells showed no differences between PBS- and DT-treated mice. Box and whisker plots display median and quartiles with maximum and minimum values. (B-E) Flow cytometric analysis of cells isolated form the brain and spleen of WT mice at $3 \mathrm{dpi}(\mathrm{B}$ and $\mathrm{C}$ ) and $7 \mathrm{dpi}(\mathrm{D}$ and $\mathrm{E})$ to determine the frequency of IFNy-producing $C D 4^{+} T$ cells ( $B$ and $D$ ) and $C D 8^{+} T$ cells $(C$ and $E)$. No significant differences were observed between PBS- and DT-treated mice. Each dot in the scatter plot represents the data from an individual mouse.

Additional file 4: Figure S3. A transient increase in the frequency of $\mathrm{CD}^{+}$and $\mathrm{CD}^{+} \mathrm{T}$ cells post TMEV infection in Treg-depleted mice. Flow cytometric analysis of cells isolated from the brain of Treg-depleted (DT) and non-depleted (PBS) mice at $3 \mathrm{dpi}$ and $7 \mathrm{dpi}$ show higher frequency of $\mathrm{CD}^{+}$and $\mathrm{CD} 8^{+} \mathrm{T}$ cells among living cells at $3 \mathrm{dpi}$ in Treg-depleted mice. ${ }^{* * *} P$-value $<0.001$.

Additional file 5: Figure S4. Effector T cells were efficiently primed in the periphery in response to TMEV infection following Treg ablation. Percentage of IFNy-producing $\mathrm{CD}^{+}$and $\mathrm{CD}^{+} \mathrm{T}$ cells in the spleen was compared between non-depleted and Treg-depleted mice at $3 \mathrm{dpi}$ and 7 dpi. ${ }^{*} P$-value $<0.05,{ }^{* * *}$-value $<0.001$.

\section{Abbreviations \\ CNS: central nervous system; MS: multiple sclerosis; DT: diphtheria toxin; DPI: days post inoculation; PVI: perivascular infiltrates; IL: interleukin; NOS2: inducible nitric oxide synthase; IP: intraperitoneal; CXCL: C-X-C chemokine ligand; CCL: C-C chemokine ligand; IFN: interferon; TNF: tumor necrosis factor; GM-CSF: granulocyte macrophage colony stimulating factor; GAPDH: glyceraldehyde 3-phosphate dehydrogenase; HPRT: hypoxanthine- guanine phosphoribosyltransferase; GFAP: glial fibrillary acidic protein; TMEV: Theiler's murine encephalomyelitis virus; TME: Theiler's murine encephalomyelitis; RT-PCR: real-time polymerase chain reaction; RNA: ribonucleic acid; mRNA: messenger ribonucleic acid; cDNA: complementary deoxyribonucleic acid.}

\section{Competing interest}

The authors declare that they have no competing interests.

\section{Authors' contributions}

$C K P, A B, J H, W B$ and $M S$ were involved in study design and data discussion. CKP, AB and MS drafted the manuscript. Experiments were performed with assistance from VG VH, IG, VH and RT. CKP performed flow cytometry, immunofluorescence and RT-PCR. AB, CDI and IG performed immunohistochemistry and RT-PCR. All authors read and approved the final manuscript.

\section{Acknowledgements}

The authors are grateful to Kerstin Schoene and Caroline Schuetz for technical assistance. We also thank Dr. Carsten Wiethe, Biolegend, for the assistance in designing the antibody staining panel for flow cytometry. This study was supported by grants from the Deutsche Forschungsgemeinschaft (STA 518/3-1 to MS, BA 815/13-1 to WB, HU 1300/3-1 to JH and BE 4200/1-2 to $A B$ ) and the Niedersachsen-Research Network on Neuroinfectiology (N-RENNT) of the Ministry of Science and Culture of Lower Saxony to WB and MS.

\section{Author details}

'Department of Neurology, Clinical Neuroimmunology and Neurochemistry, Hannover Medical School, Carl-Neuberg-Str. 1, Hannover 30625, Germany. ${ }^{2}$ Department of Pathology, University of Veterinary Medicine Hannover, Bünteweg 17, Hannover D-30559, Germany. ${ }^{3}$ Experimental Immunology, Helmholtz Centre for Infection Research, Inhoffenstr. 7, Braunschweig D-38124, Germany. ${ }^{4}$ Center of Systems Neuroscience, Hannover, Germany.

Received: 3 September 2014 Accepted: 8 October 2014

Published online: 13 November 2014

\section{References}

1. Goverman J: Autoimmune T cell responses in the central nervous system. Nat Rev Immunol 2009, 9:393-407.

2. Mayer MC, Breithaupt C, Reindl M, Schanda K, Rostasy K, Berger T, Dale RC, Brilot F, Olsson T, Jenne D, Pröbstel AK, Dornmair K, Wekerle H, Hohlfeld R, Banwell B, Bar-Or A, Meinl E: Distinction and temporal stability of conformational epitopes on myelin oligodendrocyte glycoprotein recognized by patients with different inflammatory central nervous system diseases. J Immunol 2013, 191:3594-3604.

3. Kakalacheva K, Munz C, Lunemann JD: Viral triggers of multiple sclerosis. Biochim Biophys Acta 2011, 1812:132-140.

4. Munz C, Lunemann JD, Getts MT, Miller SD: Antiviral immune responses: triggers of or triggered by autoimmunity? Nat Rev Immunol 2009, 9:246-258.

5. Miller SD, Olson JK, Croxford JL: Multiple pathways to induction of virusinduced autoimmune demyelination: lessons from Theiler's virus infection. J Autoimmun 2001, 16:219-227.

6. Lipton HL: Theiler's virus-infection in mice - unusual biphasic disease process leading to demyelination. Infect Immun 1975, 11:1147-1155.

7. Getts MT, Richards MH, Miller SD: A critical role for virus-specific CD8 (+) CTLs in protection from Theiler's virus-induced demyelination in disease-susceptible SJL mice. Virology 2010, 402:102-111.

8. Hou WQ, Kang HS, Kim BS: Th17 cells enhance viral persistence and inhibit T cell cytotoxicity in a model of chronic virus infection. J Exp Med 2009, 206:313-328.

9. Tsunoda I: Axonal degeneration as a self-destructive defense mechanism against neurotropic virus infection. Future Virol 2008, 3:579-593.

10. Tsunoda I, Fujinami RS: Two models for multiple sclerosis: experimental allergic encephalomyelitis and Theiler's murine encephalomyelitis virus. J Neuropathol Exp Neurol 1996, 55:673-686.

11. Richards MH, Getts MT, Podojil JR, Jin YH, Kim BS, Miller SD: Virus expanded regulatory $T$ cells control disease severity in the Theiler's virus mouse model of MS. J Autoimmun 2011, 36:142-154.

12. Feuerer M, Hill JA, Kretschmer K, von Boehmer H, Mathis D, Benoist C: Genomic definition of multiple ex vivo regulatory $T$ cell subphenotypes. Proc Natl Acad Sci U S A 2010, 107:5919-5924.

13. Feuerer M, Hill JA, Mathis D, Benoist C: Foxp3 (+) regulatory T cells: differentiation, specification, subphenotypes. Nat Immunol 2009, 10:689-695.

14. MacDonald AJ, Duffy M, Brady MT, McKiernan S, Hall W, Hegarty J, Curry M, Mills KHG: CD4 T helper type 1 and regulatory T cells induced against the same epitopes on the core protein in hepatitis $C$ virus-infected persons. $\mathrm{J}$ Infect Dis 2002, 185:720-727. 
15. Sakaguchi S: Regulatory $T$ cells: mediating compromises between host and parasite. Nat Immunol 2003, 4:10-11.

16. Sakaguchi S, Ono M, Setoguchi R, Yagi H, Hori S, Fehervari Z, Shimizu J, Takahashi T, Nomura T: Foxp3 (+) CD25 (+) CD4 (+) natural regulatory T cells in dominant self-tolerance and autoimmune disease. Immunol Rev 2006, 212:8-27.

17. Vignali DA: How regulatory T cells work (vol 8, pg 523, 2008). Chin Med J 2011, 124:1884-1884.

18. Herder V, Gerhauser I, Klein SK, Almeida P, Kummerfeld M, Ulrich R, Seehusen F, Rohn K, Schaudien D, Baumgartner W, Huehn J, Beineke A: Interleukin-10 expression during the acute phase is a putative prerequisite for delayed viral elimination in a murine model for multiple sclerosis. J Neuroimmunol 2012, 249:27-39.

19. Martinez NE, Karlsson F, Sato F, Kawai E, Omura S, Minagar A, Grisham MB, Tsunoda I: Protective and detrimental roles for regulatory T cells in a viral model for multiple sclerosis. Brain Pathol 2014, 24:436-451.

20. Lahl K, Loddenkemper C, Drouin C, Freyer J, Arnason J, Eberl G, Hamann A, Wagner H, Huehn J, Sparwasser T: Selective depletion of Foxp3+ regulatory T cells induces a scurfy-like disease. J Exp Med 2007, 204:57-63.

21. Nicklas W, Baneux P, Boot R, Decelle T, Deeny AA, Fumanelli M, Illgen-Wilcke B: Recommendations for the health monitoring of rodent and rabbit colonies in breeding and experimental units. Lab Anim 2002, 36:20-42.

22. Gerhauser I, Alldinger S, Baumgartner W: Ets-1 represents a pivotal transcription factor for viral clearance, inflammation, and demyelination in a mouse model of multiple sclerosis. J Neuroimmunol 2007, 188:86-94.

23. Gerhauser I, Alldinger S, Ulrich R, Baumgartner W: Spatio-temporal expression of immediate early genes in the central nervous system of SJL/J mice. Int J Dev Neurosci 2005, 23:637-649.

24. Ulrich R, Baumgartner W, Gerhauser I, Seeliger F, Haist V, Deschl U, Alldinger S: MMP-12, MMP-3, and TIMP-1 are markedly upregulated in chronic demyelinating theiler murine encephalomyelitis. J Neuropath Exp Neur 2006, 65:783-793.

25. Vandesompele J, De Preter K, Pattyn F, Poppe B, Van Roy N, De Paepe A, Speleman F: Accurate normalization of real-time quantitative RT-PCR data by geometric averaging of multiple internal control genes. Genome Biol 2002, 3. Epub 2002 Jun 18.

26. Jin $\mathrm{YH}$, Kang $\mathrm{HS}$, Mohindru M, Kim BS: Preferential induction of protective T cell responses to Theiler's virus in resistant (C57BL/6 $\times$ SJL) F1 mice. J Virol 2011, 85:3033-3040.

27. Pullen LC, Park SH, Miller SD, Dal Canto MC, Kim BS: Treatment with bacterial LPS renders genetically resistant C57BL/6 mice susceptible to Theiler's virus-induced demyelinating disease. J Immunol 1995, 155:4497-4503.

28. Lyman MA, Myoung J, Mohindru M, Kim BS: Quantitative, not qualitative, differences in CD8 (+) T cell responses to Theiler's murine encephalomyelitis virus between resistant C57BL/6 and susceptible SJL/J mice. Eur J Immuno/ 2004, 34:2730-2739.

29. McGeachy MJ, Stephens LA, Anderton SM: Natural recovery and protection from autoimmune encephalomyelitis: contribution of CD4 + CD25+ regulatory cells within the central nervous system. J Immunol 2005, 175:3025-3032.

30. Inoue A, Koh CS, Yahikozawa H, Yanagisawa N, Yagita H, Ishihara Y, Kim BS: The level of tumor necrosis factor-alpha producing cells in the spinal cord correlates with the degree of Theiler's murine encephalomyelitis virus-induced demyelinating disease. Int Immunol 1996, 8:1001-1008.

31. Pullen LC, Miller SD, Dalcanto MC, Vandermeide PH, Kim BS: Alteration in the level of interferon-gamma results in acceleration of Theiler's virus-induced demyelinating disease. J Neuroimmunol 1994, 55:143-152.

doi:10.1186/s12974-014-0180-9

Cite this article as: Prajeeth et al: Limited role of regulatory T cells during acute Theiler virus-induced encephalitis in resistant C57BL/6 mice. Journal of Neuroinflammation 2014 11:180.

\section{Submit your next manuscript to BioMed Central and take full advantage of:}

- Convenient online submission

- Thorough peer review

- No space constraints or color figure charges

- Immediate publication on acceptance

- Inclusion in PubMed, CAS, Scopus and Google Scholar

- Research which is freely available for redistribution

Submit your manuscript at www.biomedcentral.com/submit
C Biomed Central 